Revue d'histoire de l'Amérique française

सES REVUE D.HISTOIRE DE L'AMÉRIQUE FRANÇAISE

\title{
Quand la Société Saint-Jean-Baptiste a-t-elle été fondée ?
}

\section{Robert Rumilly}

Volume 1, numéro 2, septembre 1947

URI : https://id.erudit.org/iderudit/801368ar

DOI : https://doi.org/10.7202/801368ar

Aller au sommaire du numéro

Éditeur(s)

Institut d'histoire de l'Amérique française

ISSN

0035-2357 (imprimé)

1492-1383 (numérique)

Découvrir la revue

Citer cet article

Rumilly, R. (1947). Quand la Société Saint-Jean-Baptiste a-t-elle été fondée ?

Revue d'histoire de l'Amérique française, 1(2), 237-242.

https://doi.org/10.7202/801368ar d'utilisation que vous pouvez consulter en ligne.

https://apropos.erudit.org/fr/usagers/politique-dutilisation/ 


\section{QUAND LA SOCIÉTÉ SAINT-JEAN-BAPTISTE A-T-ELLE ÉTE FONDÉE?}

L'époque des 92 Résolutions - Le banquet du 24 juin 1834 - Petite discussion historique.

De longues années d'injustice, de mécontentement et d'agitation ont préparé la "Révolution" de 1837. Tous les historiens de cette période ont marqué, au passage, l'importance et le retentissement des 92 Résolutions, adoptées par la Chambre le 21 février 1834. Les 92 Résolutions rédigées dans le style emphatique de l'époque-qui était le style de Papineau - comprenaient des professions de foi démocratiques et un rappel de l'exemple américain, ce qui ressemblait beaucoup à une menace. Les griefs qu'elles exposaient, les idées qu'elles proclamaient et les débats qu'elles avaient suscités alimentèrent toutes les conversations des " patriotes ".

La Minerve formait naturellement centre de conciliabules et d'effervescence. Ludger Duvernay courait bravement le risque d'une troisième incarcération. Il fonda, au mois de mars, la Société " Aidetoi, le Ciel t'aidera " dont les membres devaient fournir, à tour de rôle, un essai sur la politique ou sur la littérature. Duvernay présidait lui-même la Société, avec Louis Perreault pour vice-président et Victor Sicotte pour secrétaire. "Aide-toi, le Ciel t'aidera " groupa de jeunes patriotes, qui parlèrent politique plus souvent que littérature. Une discussion suivait la lecture des essais, et la demande d'un seul membre obligeait au secret ${ }^{1}$. Duvernay conçut encore l'idée d'une fête annuelle, groupant les Canadiens français, et choisit le jour de la Saint-Jean-Baptiste.

1. Une communication de Montarville Boucher de La Bruère à E.-Z. Massicotte, publiée dans le Bulletin des Recherches historiques de 1928 (p. 107) fournit des renseignements tirés de papiers de famille, sur la Société "Aide-toi, le Ciel t'aidera". 
Le prénom de Jean-Baptiste était répandu parmi les Canadiens comme celui de Patrick parmi les Irlandais. Au point que le surnom de "Jean-Baptiste " s'appliquait aux Canadiens français comme celui de Patrick aux Irlandais, celui de John Bull aux Anglais et celui de Jonathan aux Américains. La fête de la Saint-Jean-Baptiste renouerait une tradition, celle des fêtes de la Saint-Jean, célébrées à Québec aux premiers temps de la colonie. Elle offrirait enfin l'avantage de tomber en plein solstice d'été, pendant les plus longs et les plus beaux jours de l'année, que l'antiquité consacrait au culte du soleil.

Il semble bien que Duvernay ait tout de suite conçu le projet d'une fête nationale. Mais il commença par organiser, pour le soir du 24 juin 1834, un banquet de notables, presque fatalement doublé d'une manifestation politique. La Minerve ne fit aucune publicité au projet.

\section{UNE MANIFESTATION POLITIQUE}

Les convives - les manifestants - se recrutèrent de vive voix. L'avocat John McDonnell, fils d'un officier écossais et de MarieAnne Picoté de Belestre - et qu'on appelait souvent Belestre-McDonnell ou McDonnell-Belestre - avait son bureau rue Saint-Vincent, a deux pas de l'atelier où s'imprimait la Minerve ${ }^{2}$. Il voisinait avec Duvernay et sympathisait avec les réformistes, ainsi que le prouvera son arrestation en 1839. Il avait hérité de sa mère une belle propriété, rue Saint-Antoine, à l'emplacement actuel de la gare Windsor. Il mit son jardin à la disposition de Ludger Duvernay, organisateur du banquet de la Saint-Jean-Baptiste. Jacques Viger, premier maire de Montréal, accepta la présidence.

Soixante convives se réunirent autour des tables, dans le jardin de l'avocat McDonnell, éclairé par des lanternes vénitiennes. C'étaient presque tous des chefs réformistes. Citons Duvernay et O'Callaghan, les deux journalistes; Ovide Perreault, qui sera tué à Saint-Denis;

2. Tous les renseignements que nous possédons sur John McDonnell sont dus aux recherches d'E.-Z. Massicotte. Ils ont fait l'objet d'une notice, insérée dans le XVe Volume des Mélanges historiques de Benjamin Sulte. C'est encore E.-Z. Massicotte qui a déterminé l'emplacement du jardin de McDonnell, où s'est tenu le banquet historique. 
Thomas-Storrow Brown, qui commandera les patriotes à SaintCharles; et trois députés, Sabrevois de Bleury, Louis-Hippolyte Lafontaine, Edouard-Etienne Rodier, tous trois partisans des 92 Résolutions.

Les toasts, la musique, les chants et les discours alternèrent. "Vingt-cinq santés sont énumérées dans le compte rendu que publia la Minerve ", écrit Benjamin Sulte, qui en omet une douzaine. Ces ( santés ) donnent le ton, indiquent le caractère de la manifestation:

" Le Peuple, source primitive de toute autorité légitime...

"La Chambre d'Assemblée du Bas-Canada, l'organe fidèle du peuple canadien.

"L'honorable Louis-Joseph Papineau, Orateur de la Chambre d'Assemblée, habile et zélé défenseur des Droits du peuple.

" Louis Bourdages, écuyer, Représentant du comté de Montmorency, premier maire de Québec, moteur des 92 Résolutions sur l'état de la province; et les 56 membres qui ont formé la glorieuse majorité qui les a votées.

" O'Connell et nos compatriotes irlandais.

"Jocelyn Waller (En silence).

"Daniel Tracey et les victimes du 21 mai (En silence).

« MM. D.-B. Viger et A.-N. Morin, nos agents en Angleterre.

" MM. Hume et Roebuck, et les membres libéraux de la Chambre des communes qui soutiennent nos intérêts.

« MM. W. L. Mackenzie et Bidwell, et les autres Représentants du Haut-Canada...

(I) Ge Gouvernement des Etats-Unis (Il excite l'admiration et l'envie de l'univers)...

Ces toasts résument des pages d'histoire. Ils affirment le principe essentiel (" Le Peuple, source de toute autorité légitime »), endossent les 92 Résolutions, glorifient Papineau, exaltent la mémoire de Jocelyn Waller, de Daniel Tracey et des victimes du 21 mai 1832, saluent le chef irlandais O'Connell, les agents de la province à Londres, les libéraux anglais qui les appuient, et les réformistes haut-canadiens groupés autour de Mackenzie. Ils rappellent à leur tour l'exemple des Etats-Unis. Le banquet du 24 juin 1834 fut bien une manifestation politique.

O'Callaghan et Rodier prirent la parole à plusieurs reprises. Un étudiant, Georges-Etienne Cartier, stagiaire au bureau de Rodier 
et membre de la Société "Aide-toi, le Ciel t'aidera ", chanta des couplets de sa composition - tout probablement $O$ Canada! mon pays, mes amours. Le maire Viger chanta trois couplets d'un auteur anonyme, évoquant " les Français dont nous descendons »:

" Ils ont frappé la tyrannie,

" Nous saurons l'abattre comme eux.

" Si le sort désignait une race ennemie,

(Veille sur nous, saint Jean, fais-nous victorieux. ")

Les participants décidèrent une célébration annuelle de la SaintJean-Baptiste, adoptée comme fête nationale. La Minerve du surlendemain fournit un compte rendu copieux. Le Canadien, de Québec, applaudit: "C'est avec attendrissement que nous avons lu le rapport d'une réunion patriotique à Montréal, à l'occasion de la fête de la Saint-Jean-Baptiste, qu'on a érigée en fête patronale... )

\section{UNE CONFUSION S'EST PRODUITE}

La tradition fait remonter la fondation de la Société Saint-JeanBaptiste de Montréal à ce banquet du 24 juin 1834. Le notaire Victor Morin, érudit et actif président de la Société, a fait poser en 1926, près de la porte d'entrée de la gare Windsor, une plaque de bronze portant cette inscription: "Site du jardin de John de Belestre-McDonnell, où la Société Saint-Jean-Baptiste fut fondée par Ludger Duvernay, le 24 juin 1834. ") La Société elle-même a célébré son centenaire en 1934.

D'après la tradition, la Société, fondée en 1834 et dispersée par les " troubles » de 1837, aurait été réorganisée en 1843.

Or nous ne connaissons d'autre source que le compte rendu de la Minerve. L.-O. Turcotte, dans un article du Journal de Québec souvent cité (23 juin 1874), et Benjamin Sulte dans ses Mélanges historiques, se sont inspirés de ce compte rendu.

La Minerve décrit longuement le banquet, destiné à fonder une fête nationale, non pas une société. Ce compte rendu et ceux de 1835, de 1836 et de 1837, tout probablement rédigés par Duvernay luimême, et très détaillés, ne mentionnent aucune organisation de société, constituée avec un bureau, des règlements, des assemblées. Deux toasts de 1834 sont ainsi conçus:

"M. Duvernay, président de la Société " Aide-toi, le Ciel t'aidera ", qui a donné l'idée de cette fête et en a surveillé les préparatifs". 
" Aide-toi, le Ciel t'aidera ". Cette société naissante, composée de l'élite de notre jeunesse, fait concevoir les plus flatteuses espérances ).

Il ne sera plus question de la Société "Aide-toi, le Ciel t'aidera " dès le banquet de 1835. Pour préparer les célébrations des années suivantes, Duvernay convoquera, par la voix de son journal, non pas les membres d'une société inexistante, mais les citoyens de bonne volonté, qui se réunissent à la librairie Fabre. Ni les convocations, ni les toasts - interminables! - ni les discours ne contiennent la moindre allusion à une Société Saint-Jean-Baptiste. Les convives de 1835 et de 1837 boivent à la santé de Ludger Duvernay, "fondateur de notre fête nationale, la Saint-Jean-Baptiste " - non pas "fondateur de notre société nationale ). Enfin les archives de la Société ne renferment aucune pièce antérieure à 1843.

Selon toute apparence, c'est par une légère confusion qu'on a parlé d'une "réorganisation" de la Société Saint-Jean-Baptiste en 1843. Il y aurait eu, en mars 1834, fondation d'une éphémère société "Aide-toi, le Ciel t'aidera " par Duvernay et ses amis; en juin 1834, fondation de la fête de la Saint-Jean-Baptiste par les mêmes personnes; en 1843, fondation de la Société Saint-Jean-Baptiste, toujours par les mêmes personnes, la Société assumant, entre autres buts, la reprise de la fête annuelle, interrompue après les " troubles"). Dans sa communication relative à la Société " Aide-toi, le Ciel t'aidera ", Montarville de La Bruère écrivait: " La Société Saint-JeanBaptiste, comme association ou société bien définie, ne devait voir le jour qu'après le retour de l'exil, en 1842, de Duvernay. ")

Empressons-nous d'ajouter que la confusion remonte aux premiers commentateurs. L.-O. Turcotte, dans son article de 1874, parle indifféremment de fête et de société:

"A l'occasion du quatrième anniversaire de la fondation de la Société Saint-Jean-Baptiste...

« La fondation de la fête nationale remonte à $1834 . .$.

" L'association, ou plutôt la société de la Saint-Jean-Baptiste, comme tout ce qui est destiné à devenir grand, eut un début très humble. M. Duvernay ne prit que deux jours pour faire les préparatifs de la fête... ")

La conscience de l'historien - ou sa déformation professionnelle - exigeait cette digression. La tradition ne s'en trouvera point 
bouleversée. Nous pouvons considérer la fondation d'“ Aide-toi, le Ciel t'aidera " comme un essai précurseur. Puis, entre la fête du 24 juin et la Société Saint-Jean-Baptiste, à défaut d'identité, la filiation est incontestable. On pourrait soutenir que la Société Saint-JeanBaptiste existait virtuellement, spontanément, dès 1834, et qu'elle a pris une forme plus ordonnée en 1843.

Robert RumILly

de l'Académie canadienne-française 